\title{
Evaluation of Integrated Disease Surveillance and Response (IDSR) using Bacillary Dysentery as a Priority Disease, Tanzania, 2012
}

\author{
Alfred G. Mwanyika*1, Senga Sembuche ${ }^{1}$ and Agricola Joachim ${ }^{2}$ \\ ${ }^{1}$ Tanzania Field Epidemiology and Laboratory Training Program (TFELTP), Dar es Salaam, United Republic of Tanzania; ${ }^{2}$ Muhimbili \\ University of Health and Allied Sciences (MUHAS), Dar es Salaam, United Republic of Tanzania
}

\section{Objective}

To determine whether the IDSR system meets its purpose and objectives, to evaluate the system attributes, and provide recommendations to improve the IDSR system, using the example of bacillary dysentery, a priority disease in Tanzania.

\section{Introduction}

Each year Ministry of Health and Social welfare of Tanzania under Epidemiology Section has been reporting many suspected cases of Shigella throughout the country. However only fewer laboratories have been reporting the confirmed cases.

\section{Methods}

The study was conducted between November 2011 and February 2012.Hospital staff including nurses, physicians and laboratory personnel and other stakeholders from the Ministry of Health and Social Welfare (MOHSW) were enrolled in the study. Data was collected from a review of documents, questionnaires and interview of stakeholders. Surveillance system attributes were evaluated using updated guidelines for evaluating public health surveillance system (2007) from Morbidity and Mortality Weekly Report (MMWR).

\section{Results}

Questionnaires were administered to fifteen health personnel from four regional hospitals. Four health staff from epidemiology and laboratory section of MOHSW were interviewed. Only one regional hospital laboratory was conducting laboratory diagnosis for bacillary dysentery and sending reports to MOHSW. Data from this laboratory was reviewed. Out of 641 records from bacillary dysentery testing, $271(42.3 \%)$ did not include age data, $5(0.78 \%)$ missed sex, 624 $(97.3 \%)$ missed the district where the patient came from, $26(4.4 \%)$ did not include information on specimen quality, $1(0.2 \%)$ had no report of pathogens found and $636(99.2 \%)$ did not include antimicrobial sensitivity testing (AST). The Predictive Value Positive (PVP) of the system was $0.62 \%$. One $(6.7 \%)$ of the health workers was trained in IDSR.

\section{Conclusions}

IDSR in Tanzania generally is not performing well as only one $(25 \%)$ of the four visited hospitals conducts and reports laboratory diagnosis of bacillary dysentery. However the system is representative as it covers all regions of the United Republic of Tanzania and all ages of people. The system is flexible since National IDSR guideline (2001) was revised in 2011. More emphasis should be placed on strengthening laboratory capacity in disease diagnosis and reporting at all levels.

\section{Keywords}

Surveillance; Evaluation; IDSR; Bacillary dysentery

\section{Acknowledgments}

We acknowledge TFELTP and regional hospitals for their corporations to achieve this study.

\section{References}

Monthly diseases reports. Ministry of health and social welfare of Tanzania. www.moh.go.tz

*Alfred G. Mwanyika

E-mail: geofalfred@yahoo.com 